\title{
Prediction of Concrete Corrosion in Sulfuric Acid by SVM-Based Method
}

\author{
Weihang Zhang ${ }^{1, a}$, Zhigang Song ${ }^{1, b}$ \\ ${ }^{1}$ Faculty of Civil Engineering, Kunming University of Science and Technology, Kunming, P.R. China, \\ 650500 \\ alibrahang@gmail.com, bzhigang-s@163.com
}

Keywords: support vector machine; concrete; sulfuric acid; corrosion model

\begin{abstract}
A long time immersion test of fly ash concrete specimens in sulfuric acid is carried out. On the basis of the experimental datum, a novel prediction model for sulfuric acid corrosion of concrete based on support vector machine (SVM) is proposed. The design steps and learning algorithm are also given. Five main influencing factors including the water and cement ratio, $\mathrm{pH}$ value of the soaking solution, cement and fly ash quantity, specimens fluidity are analyzed with the proposed model. By comparison with the measured results, the mean square error (MSE) of predicted result of the model is 0.188 . It implies that this new method of computation appears to be a useful tool for sulfuric acid corrosion of fly ash concrete research.
\end{abstract}

\section{Introduction}

Sulfuric acid is one of the corrosion media to concrete. Due to its complex corrosion law and multiple influencing parameters [1,2], there is no effective prediction method right now. One feasible way to overcome this problem is to construct a corrosion database with prediction function basing on experiments, and then the prediction model can be refined step by step with the accumulation of experiment datum. However, the requirements of least square sum of residuals in traditional regression methods generally involve the error in the limited sample data and may cause over fitting problem. In the condition of more variables with fewer observed samples, the problem is particularly severe. Support vector machines (SVM), a new method proposed by Vapnik is widely adopted in recent years for its effectiveness in processing nonlinear datum of small sample while avoiding the over fitting problem.

A long time immersion test of fly ash concrete specimens in sulfuric acid is carried out in this study. On the basis of the experimental datum, the sulfuric acid corrosion database is established, and the prediction model of sulfuric acid corroding fly ash concrete based on SVM is proposed. Five main factors influencing the reaction rate, i.e. water-cement ratio, $\mathrm{pH}$ value of the soaking solution, cement and fly ash quantity and mixture fluidity, are analyzed with this model. By comparison with the measured results, the SVM model's precision of prediction is obtained.

\section{The Experiment}

There are totally 30 groups of tests in the experiment study and the detail information of each tests is shown in Table 1. The chemical compositions of cement and fly ash are shown in Table 2 .

Table 1 Mix proportion and $\mathrm{pH}$ value of solution

\begin{tabular}{|c|c|c|c|c|c|c|c|}
\hline \multirow{2}{*}{ Group } & \multirow{2}{*}{$m_{w} / m_{b}$} & \multicolumn{3}{|c|}{ Mix proportion(by mass ratio) } & \multirow{2}{*}{$\begin{array}{c}\text { Fluidity } \\
/ \mathrm{mm}\end{array}$} & \multirow{2}{*}{$\mathrm{pH}$} \\
\cline { 3 - 7 } & & Cement & Fly ash & Sand & Gravel & & 4.6 \\
\hline 1 & 0.53 & 1.775 & 1.395 & 4.975 & 8.470 & 36 & 4.6 \\
\hline 2 & 0.66 & 2.342 & 0.204 & 5.681 & 8.702 & 36 & 4.4 \\
\hline 3 & 0.47 & 2.717 & 0.858 & 4.510 & 8.755 & 36 & 4.4 \\
\hline 4 & 0.59 & 1.310 & 1.538 & 5.200 & 8.484 & 30 & 4.2 \\
\hline 5 & 0.43 & 2.500 & 1.407 & 4.348 & 8.441 & 31 & 4.2 \\
\hline 6 & 0.64 & 1.838 & 0.788 & 5.719 & 8.401 & 55 & 4.2 \\
\hline
\end{tabular}




\begin{tabular}{|c|c|c|c|c|c|c|c|}
\hline 7 & 0.51 & 3.162 & 0.132 & 4.949 & 8.798 & 31 & 4.0 \\
\hline 8 & 0.69 & 1.217 & 1.217 & 5.801 & 8.348 & 60 & 4.0 \\
\hline 9 & 0.56 & 2.400 & 0.600 & 5.058 & 8.799 & 38 & 3.8 \\
\hline 10 & 0.46 & 1.534 & 2.118 & 4.284 & 8.503 & 35 & 3.8 \\
\hline 11 & 0.58 & 2.433 & 0.463 & 5.246 & 8.744 & 36 & 3.6 \\
\hline 12 & 0.61 & 1.818 & 0.936 & 5.444 & 8.516 & 30 & 3.6 \\
\hline 13 & 0.41 & 3.606 & 0.492 & 4.263 & 8.656 & 32 & 3.4 \\
\hline 14 & 0.67 & 1.504 & 1.003 & 5.662 & 8.494 & 43 & 3.4 \\
\hline 15 & 0.50 & 1.747 & 1.613 & 4.689 & 8.519 & 55 & 3.2 \\
\hline 16 & 0.63 & 2.613 & 0.053 & 5.729 & 8.593 & 50 & 3.2 \\
\hline 17 & 0.44 & 2.749 & 1.069 & 4.477 & 8.500 & 46 & 3.0 \\
\hline 18 & 0.55 & 1.222 & 1.833 & 4.827 & 8.581 & 53 & 3.0 \\
\hline 19 & 0.70 & 1.776 & 0.624 & 5.964 & 8.407 & 40 & 2.8 \\
\hline 20 & 0.49 & 2.811 & 0.617 & 4.715 & 8.756 & 35 & 2.8 \\
\hline 21 & 0.62 & 1.463 & 1.246 & 5.490 & 8.408 & 50 & 2.6 \\
\hline 22 & 0.54 & 2.800 & 0.311 & 5.193 & 8.655 & 55 & 2.6 \\
\hline 23 & 0.42 & 1.920 & 2.080 & 4.184 & 8.305 & 46 & 2.4 \\
\hline 24 & 0.57 & 1.827 & 1.120 & 5.081 & 8.652 & 49 & 2.4 \\
\hline 25 & 0.68 & 2.125 & 0.346 & 5.832 & 8.569 & 47 & 2.2 \\
\hline 26 & 0.52 & 2.197 & 1.034 & 4.931 & 8.578 & 35 & 2.2 \\
\hline 27 & 0.45 & 3.509 & 0.224 & 4.399 & 8.932 & 46 & 2.0 \\
\hline 28 & 0.65 & 1.137 & 1.447 & 5.439 & 8.507 & 50 & 2.0 \\
\hline 29 & 0.48 & 2.030 & 1.470 & 4.530 & 8.601 & 35 & 1.8 \\
\hline 30 & 0.60 & 2.184 & 0.616 & 5.399 & 8.625 & 33 & 1.8 \\
\hline
\end{tabular}

Table 2 Chemical composition of cement and fly ash

\begin{tabular}{|c|c|c|c|c|c|c|}
\hline Material & $\mathrm{SiO}_{2}$ & $\mathrm{Fe}_{2} \mathrm{O}_{3}$ & $\mathrm{Al}_{2} \mathrm{O}_{3}$ & $\mathrm{CaO}$ & $\mathrm{MgO}$ & $\mathrm{SO}_{3}$ \\
\hline Cement & 24.3 & 4.8 & 3.8 & 55.3 & 4.2 & 2.4 \\
\hline Fly ash & 52.7 & 9.7 & 25.8 & 3.7 & 1.2 & 5.0 \\
\hline
\end{tabular}

Fluidity of mixture is measured according to Ref. [3]. The specimens are prepared according to Ref. [4]. After the cast of specimens, they are put in standard curing box (constant temperature $20^{\circ} \mathrm{C}$, relative humidity $95 \%$ ) for 24 hours, and then put in saturated sodium hydroxide solution. 28 days later, they are naturally dried in the room for 7 days and then cleaned to remove the surface calcium hydroxide with a metal brush. 5 surfaces of each concrete cube are waxed and only 1 surface left for soaking. After waxed, the test groups, 3 specimens for each, are put into the plastic soaking boxes with a size of $467 \mathrm{~mm} \times 363 \mathrm{~mm} \times 215 \mathrm{~mm}$.

The $\mathrm{pH}$ change of solution is monitored through a portable $\mathrm{pH}$ meter, model $\mathrm{pHB}-1$ with an accuracy of $\pm 0.01 \mathrm{pH}$ unit. Titration sulfuric acid with concentration of $0.125 \mathrm{~mol} / \mathrm{L}$ or $0.5 \mathrm{~mol} / \mathrm{L}$ is added into the soaking solution to keep the original $\mathrm{pH}$ value of soaking solution. The apparent diffusion coefficient value $\left(D_{\mathrm{OH}}\right)$ of concrete is analyzed through an analytical expression by Song et al [5], see Table 3 .

Table 3 Experiment result

\begin{tabular}{|c|c||c|c||c|c|}
\hline Group & $\ln D_{O H} \times 10^{-10}$ & Group & $\ln D_{O H} \times 10^{-10}$ & Group & $\ln D_{O H} \times 10^{-10}$ \\
\hline 1 & 1.72 & 11 & 0.62 & 21 & -4.36 \\
\hline 2 & 0.35 & 12 & 0.01 & 22 & 4.27 \\
\hline 3 & 0.89 & 13 & -0.96 & 23 & 3.33 \\
\hline 4 & 1.27 & 14 & -0.11 & 24 & -4.14 \\
\hline 5 & -1.44 & 15 & 0.86 & 25 & 6.87 \\
\hline 6 & -0.41 & 16 & -0.21 & 26 & 6.98 \\
\hline 7 & 1.53 & 17 & 1.88 & 27 & 6.31 \\
\hline 8 & 1.11 & 18 & -6.36 & 28 & 5.86 \\
\hline 9 & -0.97 & 19 & 2.67 & 29 & 5.46 \\
\hline
\end{tabular}




\begin{tabular}{|l|l||l|l||l|l|}
\hline 10 & 0.57 & 20 & 2.32 & 30 & 8.14 \\
\hline
\end{tabular}

\section{Support Vector Machine}

SVM is based on statistical learning theory developed by Vapnik [6]. It is effective to improve generalization performance and can obtain globally optimal and unique solution. It is generally used for pattern recognition and regression estimation. In SVM regression, the basic objective is to minimize an upper bound of the generalization error based on the structural risk minimization (SRM). The detailed description of SVM can be found in the publications by Vapnik [7].

The main idea of SVM regression is to map the input data $x$ into a high-dimensional feature space by a nonlinear mapping and to do linear regression in this space. The regression model is defined as $y$ $=f(x)+e$, where $x$ and $y$ are input and output function, respectively, and defined in the high-dimensional feature space; $e$ is the independently random error.

Given a dataset $G=\left\{\left(x_{i}, y_{i}\right), i=1,2 \ldots N\right\}$, where $N$ is the size of the training data set, $x_{i}$ is the value of the input and $y_{i}$ is the value of output. The optimum regression function can be assumed as follows:

$f(x)=w \varphi(x)+b$.

Where $\varphi(x)$ represents the high-dimensional feature spaces which are nonlinearly mapped from the input space $x, w$ is the weight vector and $b$ is the scalar threshold. Introducing the $\varepsilon$-insensitive loss function

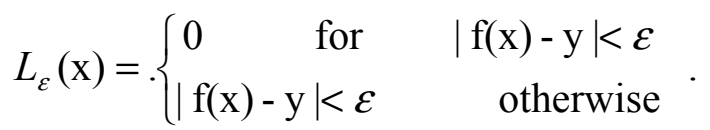

The Eq. 1 can be transformed to the dual optimization problem:

$$
\max _{\alpha, \alpha^{*}} \mathrm{~W}\left(\alpha, \alpha^{*}\right)=\max _{\alpha, \alpha^{*}}\left\{\begin{array}{c}
\sum_{i=1}^{N} \alpha_{i}^{*}\left(x_{i}-\varepsilon\right)-\alpha_{i}\left(x_{i}+\varepsilon\right) \\
-\frac{1}{2} \sum_{i=1, j=1}^{N}\left(\alpha_{i}^{*}-\alpha_{i}\right)\left(\alpha_{j}^{*}-\alpha_{j}\right) \varphi\left(\mathrm{x}_{\mathrm{i}}, \mathrm{x}_{\mathrm{j}}\right)-\varepsilon \sum_{i=1}^{N}\left(\alpha_{i}^{*}-\alpha_{i}\right)+\sum_{i=1}^{N} \mathrm{y}_{\mathrm{i}}\left(\alpha_{i}^{*}-\alpha_{i}\right)
\end{array}\right.
$$

Where $0 \leq \alpha_{i} \leq C, 0 \leq \alpha_{i}^{*} \leq C$ and $\sum_{i=1}^{N}\left(\alpha_{i}^{*}-\alpha_{i}\right)=0$. The Eq. 3 can be solved by Lagrangian multipliers, and its solution is given by:

$$
f(\mathrm{x})=\sum_{i=1}^{N}\left(\alpha_{i}-\alpha_{i}^{*}\right) \varphi\left(\mathrm{x}_{\mathrm{i}}, \mathrm{x}_{\mathrm{j}}\right)+\mathrm{b} .
$$

The kernel function $K\left(x_{i}, x_{j}\right)$ has been introduced to avoid computing explicitly the map $\varphi(x)$ [8]. Thus, Eq. 4 can be written as:

$$
f(\mathrm{x})=\sum_{i=1}^{N}\left(\alpha_{i}-\alpha_{i}^{*}\right) K\left(\mathrm{x}_{\mathrm{i}}, \mathrm{x}_{\mathrm{j}}\right)+\mathrm{b} .
$$

\section{SVM for prediction of sulfuric acid corroding concrete}

This study attempts to use SVM for the prediction of sulfuric acid corroding concrete. The experimental datum and results (see Table 2 and 3 ) are used in this study.

First, choosing 1 out of 26 groups for training data set, the rest are considered as testing data set. All the input data (water-cement ratio, $\mathrm{pH}$, cement quantity, fly ash quantity, mixture fluidity) and output data $\left(D_{O H}\right)$ are normalized within the program (step1 and 2). Then training patterns are selected and transformed into feature space using kernel function (step 3). By using the LIBSVM [9], the predicted results are obtained, and then validated using the testing set (step 4). The predicted results are compared with the experimental results (step 5).

A problem regarding the choice of two parameters $(C$ and $\sigma$ ) for SVM was studied by Cherkassky [10]. The parameter $C$ controls the smoothness or flatness of the approximate function. The Gaussian radial basis kernel was chosen for the SVM because it is the most common and successfully applied kernel [11]. $\sigma$ is the parameter that determines the spread of the Gaussian function in the Gaussian 
radial basis kernel. $\mathrm{K}$-fold cross validation is used in this study to determine the best values of the training parameters $C$ and $\sigma$. The values of $C$ and $\sigma$ are 16 and 1.18 .

For convenient comparison purpose, the experimental and predicted results are plotted in Fig. 1

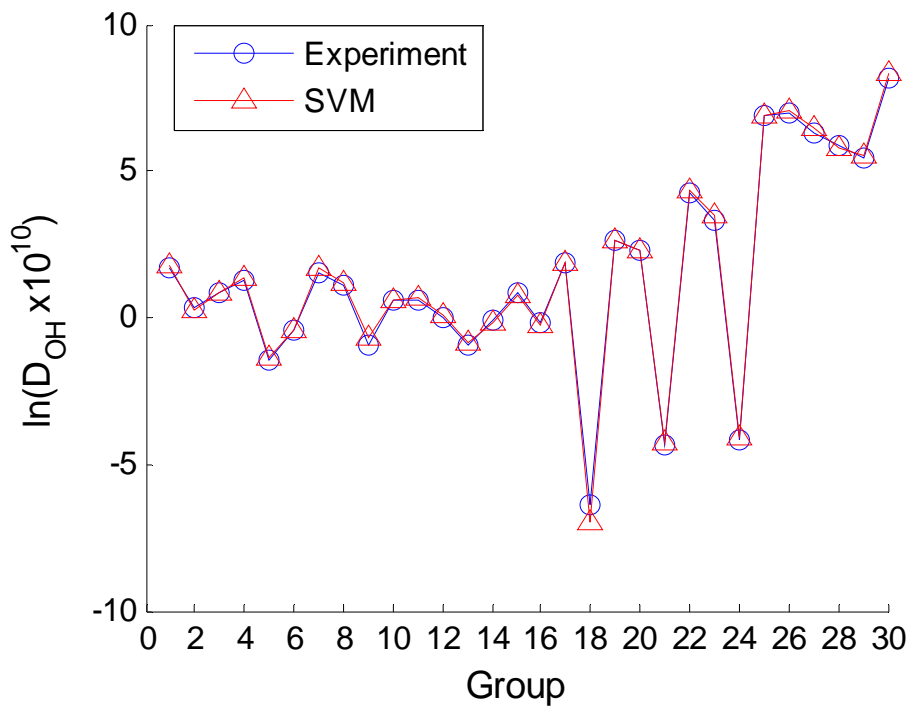

Fig.1 Comparison of experimental results with SVM

The mean squares error (MSE) is used as the main criteria to evaluate the performance of the SVM model. It is only 0.188 .

\section{Conclusions}

This study has investigated the use of SVM for prediction of sulfuric acid corroding concrete. The performance of the proposed method is verified by comparing the predicted results with the experimental results. MSE of the predicted results by SVM method is only 0.188 . It implies that SVM can be regarded as a very effective method for prediction of sulfuric acid corroding concrete.

\section{Acknowledgements}

The study of this paper is supported by the Natural Science Foundation of China and Yunnan Province (Grant No. 51078175, 2008E038M).

\section{References}

[1] B.E. Robin, W.D. Horst. Modelling Acid Attack on Concrete (Part I): The Essential Mechanisms. Cement and Concrete Research, 2005, 35(12): 2333-2339

[2] E. Hewayde, M. Nehdi, E.N Allouche. Experimental Investigations of the Effect of Selected Admixtures on the Resistance of Concrete to Sulfuric Acid Attack//Proceeding of International on Pipeline Engineering and Construction .Baltimore; ASCE, 2003:504-513.

[3] JGJ 55-2011, Standard for Test Method of Performance on Ordinary Fresh Concrete [S]. (In Chinese)

[4] GB/T 50080-2002, Specification for Mix Proportion Design of Ordinary Concrete [S]. (In Chinese)

[5] Song Zhigang, Zhang Xuesong. Experimental Study of Mortar under Corrosion in Sulfuric Acid [J].Journal of Building Materials, 2012(15):163-167. (In Chinese)

[6] V.N. Vapnik. The Nature of Statistical Learning Theory. Berlin: Springer, 1995.

[7] V.N. Vapnik. An Overview of Statistical Learning Theory. IEEE Transaction Neural Networks, 1999, 10(5):988-999.

[8] C. Nello, John Shawe-Taylor. An Introduction to Support Vector Machine and Other Kernel-based Learning Methods. England: Cambridge University Press, 2000. 
[9] Chih-Chung Chang, Chih-Jen Lin, LIBSVM: A Library for Support Vector Machines, 2001. Software available at http://www.csie.ntu.edu.tw/ cjlin/libsvm

[10]V. Cherkassky, Y. Ma. Practical Selection of SVM Parameters and Noise Estimation for SVM Regression. Neural Network, 2004(17):113-126.

[11]Keerthi S, Lin C.-J. Asymptotic Behaviors of Support Vector Machines with Gaussian Kernel . Neural Computation, 2003(15):1667-1689. 